\title{
Altered Expression of Nicotinic Receptors in Perinatal Life Related to Prenatal Exposure to Toxics-An Overview of the Research Carried Out on This Topic at the "Lino Rossi" Research Center of the Milan University
}

\author{
Anna Maria Lavezzi \\ "Lino Rossi" Research Center of the Milan University, Milan, Italy \\ Email: anna.lavezzi@unimi.it
}

How to cite this paper: Lavezzi, A.M. (2020) Altered Expression of Nicotinic Receptors in Perinatal Life Related to Prenatal Exposure to Toxics-An Overview of the Research Carried Out on This Topic at the "Lino Rossi" Research Center of the Milan University. Journal of Behavioral and Brain Science, 10, 420-431.

https://doi.org/10.4236/jbbs.2020.1010027

Received: January 31, 2020

Accepted: October 25, 2020

Published: October 28, 2020

Copyright $\odot 2020$ by author(s) and Scientific Research Publishing Inc. This work is licensed under the Creative Commons Attribution International License (CC BY 4.0).

http://creativecommons.org/licenses/by/4.0/

\begin{abstract}
The article aims to underline the impact of nicotine and pesticides on neuronal $\alpha 7$-nicotinic acetylcholine receptors expression in brainstem regions receiving cholinergic projections, given their fundamental role during the neuronal development. The in-depth histopathological/immunohistochemical examination of the autonomic nervous system performed at the "Lino Rossi" Research Center of the Milan University on a wide group of sudden unexpected fetal and infant deaths, highlighted the frequent hypodevelopment of brainstem structures checking the vital functions associated to altered expression of $\alpha 7$-nicotinic acetylcholine receptors and smoke absorption in pregnancy. A dysregulation of the catecholamine system was also observed in the cerebellar cortex of the same cases. However, in a not negligible percentage of sudden deaths with altered expression of $\alpha 7$-nicotinic receptors, the mothers never smoked but lived in rural areas. Specific analytical procedures showed the presence of agricultural pesticides in cerebral cortex samples of these victims. Therefore, it is possible to believe that the exposition to pesticides during pregnancy can produce the same harmful effects as nicotine on the nicotinic acetylcholine receptors. Moreover, alterations of $\alpha 7$-nicotinic acetylcholine receptors receptor expression were also detected in the lungs of many sudden perinatal death victims, allowing to consider even these findings as possible consequence of maternal exposure to toxic factors.
\end{abstract}

\section{Keywords}

Nicotine, Pesticides, Endocrine Disruptor Compounds (EDCs), $\alpha 7$-Nicotinic Acetylcholine Receptors, Neuropathology, Sudden Infant Death Syndrome 
(SIDS), Sudden Intrauterine Unexplained Death (SIUDS)

\section{Introduction}

In this paper I would like to present and discuss the main findings obtained in many years of research carried out at the "Lino Rossi Research Center for the study and prevention of the unexpected perinatal death and Sudden Infant Death Syndrome (SIDS)" of the Milan University (Italy) on a wide group of sudden fetal and infant deaths, related to alterations of the $\alpha 7$-nicotinic acetylcholine receptors expression as consequence of prenatal exposure to toxics.

I start from a premise: the "Lino Rossi" Research Center is the national referral center for the application of the Italian Law no. 31/2006 "Regulations for diagnostic post mortem investigation in victims of Sudden Infant Death Syndrome (SIDS) and Sudden Intrauterine Unexplained Death Syndrome (SIUDS)" [1]. This law states that all infants dying suddenly of suspected SIDS within the first year of age, as well as all fetuses who died after the $25^{\text {th }}$ week of gestation without any apparent cause in Italian regions, must undergo an in-depth post mortem examination, in accordance with specific guidelines, as reported below.

\section{Methods}

Overall, since 2006, the year of entry into force of the aforementioned law, around 200 cases of SIDS and 300 of SIUDS have been examined at the Lino Rossi Research Center. The applied pathological protocol was mainly focused on the in-depth examination of the autonomic nervous system [2] [3] [4] [5].

For every case, a complete medical history was collected, including information related to the main potential risk factors (especially maternal smoking, maternal alcohol and drug abuse before and during pregnancy and, when available, data on air pollution in the mother's area of residence). The mothers were asked in particular to complete a questionnaire on their smoking habits with details regarding the number of cigarettes smoked before, during and after pregnancy. The negative responses were validated by toxicological analyses on fetal and infant hair in order to search for traces of cotinine, which is a highly stable metabolite of nicotine.

\subsection{Neuropathological Procedure for the Examination of the Brainstem and Cerebellum in SIUDS/SIDS}

Figure 1 shows a scheme of the methodology for the brainstem examination. At the right, the sampling of three specimens is shown. The first specimen, ponto-mesencephalic, includes the upper third of the pons and the adjacent portion of caudal mesencephalon. The second extends from the upper portion of the medulla oblongata to the adjacent caudal portion of the pons. The third specimen includes the obex. A fourth specimen is taken from the rostral tract of 


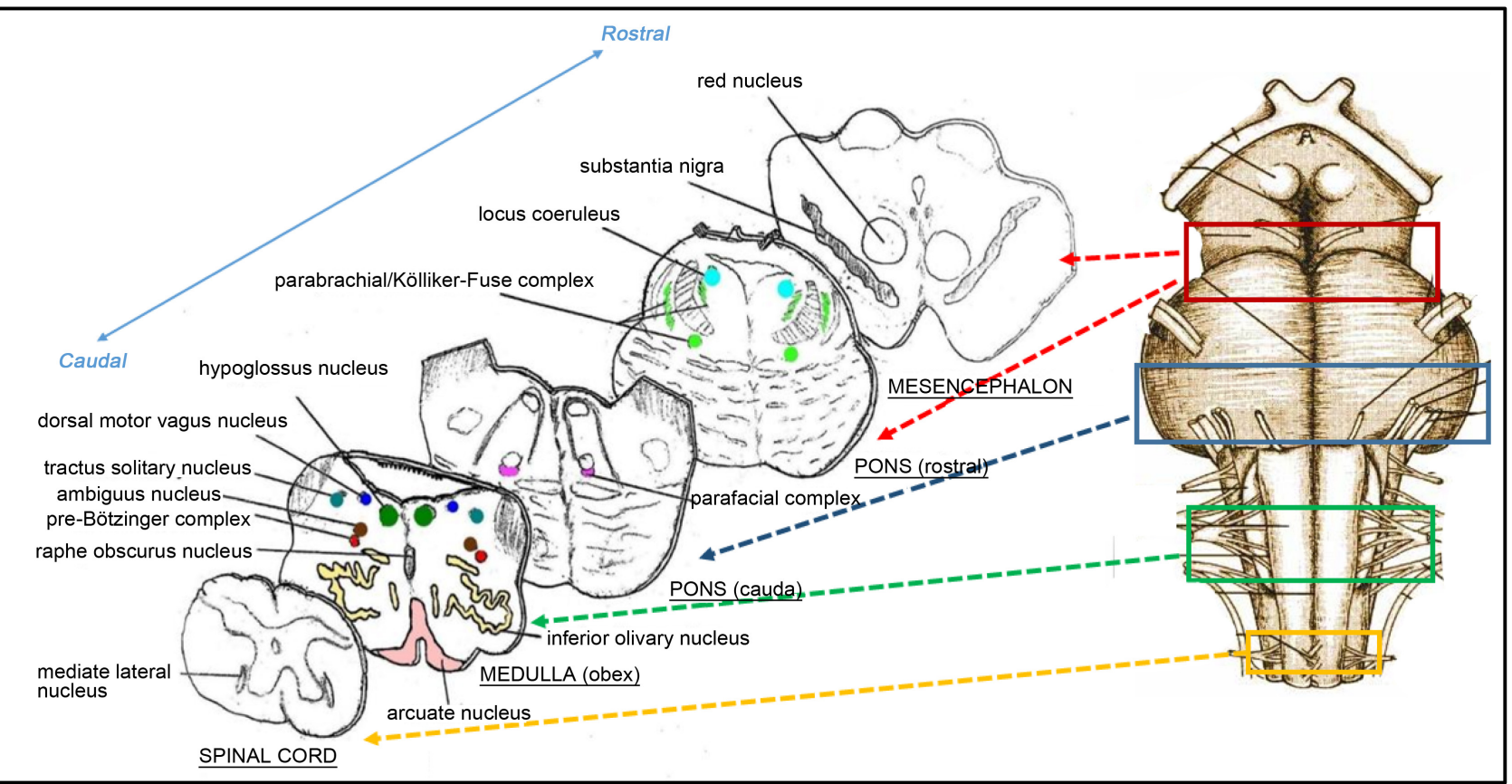

Figure 1. At the right, schematic representation of the sampling from the brainstem. At the left, the histological sections obtained from the specimens, with the indication of the main nuclei and structures to be examined.

spinal cord.

Transverse serial sections of each specimen are made at intervals of $60 \mu \mathrm{m}$. For each level, eight to ten 5 - $\mu$ m-thick sections are obtained, two of which are stained using hematoxylin-eosin and Klüver-Barrera for histological examination, by using a light microscope with a system of objective lenses to progressively magnify the images. The brainstem structures that can be recognized are: the hypoglossus, dorsal motor vagal, tractus solitarii, ambiguus, inferior olivary, pre-Bötzinger, arcuate, dorsal and ventral cochlear, medial inferior vestibular, obscurus and pallidus raphé nuclei in the medulla oblongata; the locus coeruleus, facial/parafacial complex, retrotrapezoid nucleus, superior olivary complex, superior and lateral vestibular nuclei, Kölliker-Fuse, median and magnus raphé nuclei in the pons; the inferior colliculus, substantia nigra, dorsal and caudal linear raphé nuclei in the caudal mesencephalon. In the spinal cord the intermediolateral nucleus is of great interest.

Figure 1, at the left, shows the representative histological sections obtained from the four specimens, with the indication of the most important nuclei and structures to be examined, given their frequent involvement in SIUDS/SIDS, in terms of delayed development (hypoplasia/agenesis) or malformations.

The cerebellum is excised from the brainstem by cutting the peduncles. Then, transversal samples of $60 \mu \mathrm{m}$ of both cerebellar hemispheres, including all the lobules (anterior, posterior and flocculonodular lobule) are obtained. The microscopic analysis of the cerebellum included the examination of the cortex layers, i.e. the external granular layer (EGL), molecular layer (ML), Purkinje cell layer (PCL), internal granular layer (IGL) and the medullary deep nuclei (den- 
tate nucleus, fastigial nucleus, globose nucleus and emboliform nucleus).

\subsubsection{Immunohistochemical Protocol}

The remaining sections obtained from each sample are treated, according to the needs, with specific immunohistochemical techniques in order to evaluate the expression of functional markers, whose altered expression can represent a very significant finding in SIUDS/SIDS. Among these markers are worthy of mention: the neuronal nuclear antigen, the nicotinic acetylcholine receptors, the serotonin, the somatostatin and the orexin. Here we report the methodology only related to the nicotinic receptors, and in particular to the $\alpha 7$ nicotinic receptors, as this article wants to focus on the importance of these receptors in the perinatal life and on the damage that their altered expression can cause.

\subsection{2. $\alpha$ 7-nAChR Immunohistochemistry}

The expression of $\alpha 7$ nicotinic receptors is evaluated by using a specific rabbit polyclonal antibody (aa 22-71, Abcam Ltd., UK, cod. ab10096) on the selected histological transverse sections. After dewaxing and rehydration, sections are immersed and boiled in TRIS-EDTA Buffer for antigen retrieval with a microwave oven, after blocking endogenous peroxidase by $3 \%$ hydrogen peroxide treatment. Then, sections are incubated with diluted 1:500 primary antibody overnight in a wet chamber. Samples are washed with PBS buffer and incubated with a biotinylated goat anti-rabbit IgG secondary antibody (PK-6101, Vector Laboratories, CA, USA) and then processed with the avidin-biotin-immunoperoxidase technique (VEDH-4000, Vector Laboratories, CA, USA). Finally, each section is counterstained with Mayer's Hematoxylin.

nAChR immunohistochemistry quantification-The degree of immunoreactivity is evaluated in each selected nucleus and/or structure in the brainstem and cerebellum as the number of neuronal cells showing a dark brown color, divided by the total number of neurons, and expressed as percentage (nAChR immunopositivity index: nAChR-I). The nAChR-I is classified as: "Class 0" for no or light staining (negativity); "Class 1" when the index is $<10 \%$ (weak positivity); "Class 2" with a percentage of immunopositive cells ranging between 10 and $40 \%$ (moderate positivity); "Class 3 " with an index of $>40 \%$ of the counted cells (strong positivity). Figure 2 shows many $a 7$-nAChR immunopositive neurons in the brainstem.

\subsection{Procedure for the Examination of the Lungs in SIUDS/SIDS}

Samples are obtained from each lung lobe by cutting parallel to the frontal plane and passing through the hilus. The histological examination, performed on the routinely stained sections by hematoxylin-eosin, includes the radial alveolar count (RAC), a reliable index of lung maturation closely related to the gestational age for fetuses and to postnatal age for newborns. RAC is based on the evaluation of the number of airspaces cut by a straight line drawn from the most peripheral bronchiole to the nearest connective tissue septum or pleura border. The 


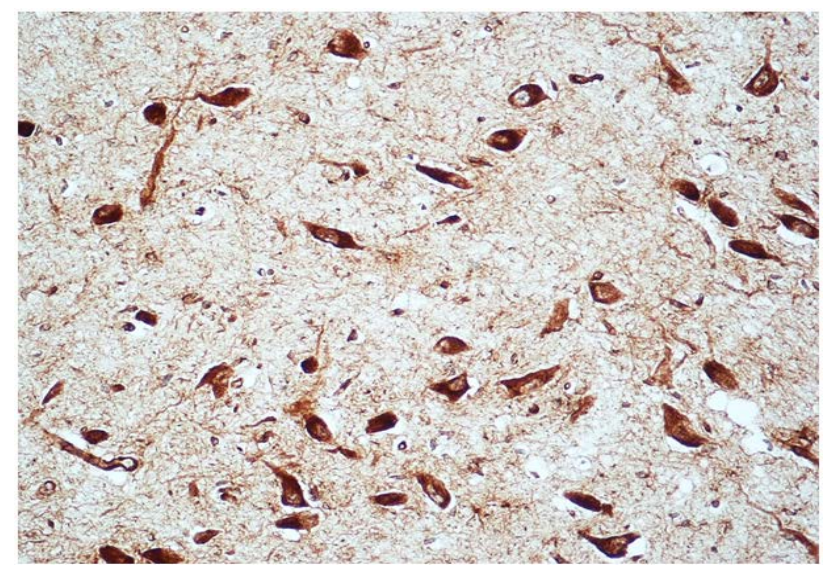

Figure 2. Strong $\alpha 7$-nAChR immunopositivity ("Class 3 ” of $\alpha 7$-nAChR-Index) in the brainstem of a SIDS case (3 month-old). Magnification: 40x.

same immunohistochemical procedure for the evaluation of the $\alpha 7$ nicotinic receptor expression in the nervous system is applicable also for the lungs. Figure 3 shows many $\alpha 7$-immunopositive nAChR neurons in the lung parenchyma.

\subsection{Procedures for the Toxicological Examination in SIUDS/SIDS}

\section{Protocol for the Chemical Characterization}

Each brain sample (approximately $0.5 \mathrm{~g}$ ) is homogenized with $2 \mathrm{~mL}$ of $n$-hexane to obtain a dense, reach supernatant. The homogenized tissue is transferred into a solid phase extraction (SPE) cartridge containing $500 \mathrm{mg}$ of $\mathrm{C}^{-}{ }_{18}$ sorbent, in order to retain most of the matrix impurities and to release the compounds of interest with hexane (34). The SPE cartridge is conditioned with $4 \mathrm{~mL}$ of $n$-hexane, before purification step, and then washed with $1 \mathrm{~mL}$ of $n$-hexane followed by $1 \mathrm{~mL}$ of dichloromethane after elution step. The extraction method is developed and validated in terms of accuracy, precision, limit of quantification (LOQ), limit of detection (LOD), and linearity. Nine isotopically labeled internal standards (ISTDs) are used for method validation.

1) Chemicals and materials: a mixture of 20 organochlorine compounds (EPA CLP mix), chlorpyrifos, chlorfenvinfos, captan, boscalid and bisphenol are purchased from Sigma-Aldrich (Milan, Italy). All solvents used ( $n$-hexane and dichloromethane) are supplied from Merk (Suprasolv, 99\% purity, Merk, Germany). Stock solutions are prepared in $n$-hexane at a concentration of 100 $\mu \mathrm{g} / \mathrm{mL}$. A standard mixture containing all compounds (25 specific EDCs and 9 ISTD) was prepared by appropriate dilution and stored at $4^{\circ} \mathrm{C}$ in the dark. SPE cartridges HyperSep-C18 $(500 \mathrm{mg} / 6 \mathrm{~mL})$ are purchased by Thermo Scientific (Bellefonte, USA).

2) Instrumentations: the analyses on the extracted samples are performed by an Agilent Technologies gas chromatograph $6890 \mathrm{~N}$, equipped with a single quadrupole mass spectrometer 5975C TAD/MS, working in electron ionization. All brain tissues are subjected to analytical procedure in order to determine the 


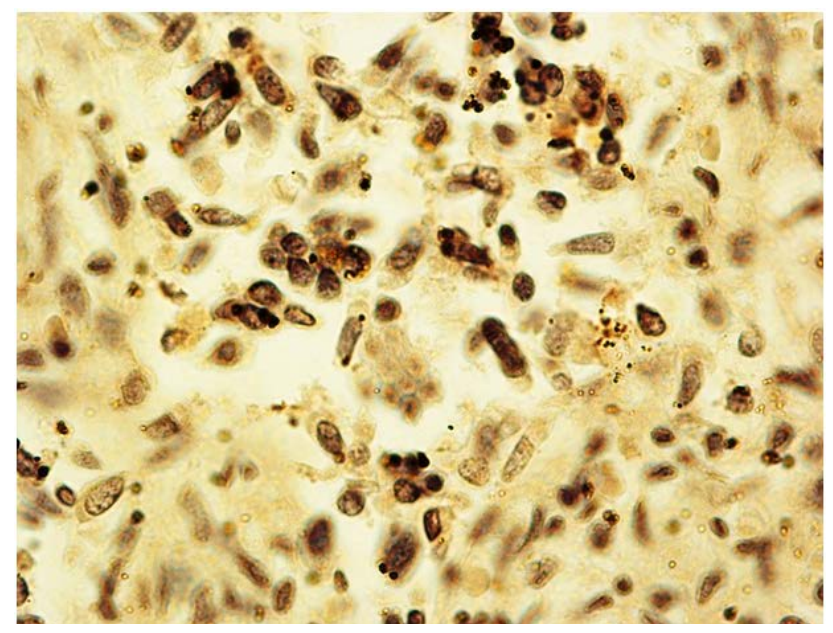

Figure 3. Positive $\alpha 7 \mathrm{nAChR}$ immunoexpression of a group of cells in the lung parenchyma of a SIUDS case (38 gestational weeks). Magnification: $40 \times$.

level of the 25 selected compounds. The chromatographic separation is carried out using an HP-5MS (Agilent J\&W GC columns, Folsom, CA, USA), i.d. $30.0 \mathrm{~m}$ $\times 0.25 \mathrm{~mm}$, containing $5 \%$ phenyl-methylsiloxane, with a phase thickness of 0.25 $\mu \mathrm{m}$. As carrier gas, helium at $1 \mathrm{~mL} / \mathrm{min}$ (constant flow) is adopted. The GC oven temperatures are programmed as follows: $80^{\circ} \mathrm{C}$ held for $1 \mathrm{~min}$, ramped at $30^{\circ} \mathrm{C} / \mathrm{min}$ to $180^{\circ} \mathrm{C}$, ramped at $3^{\circ} \mathrm{C} / \mathrm{min}$ to $225^{\circ} \mathrm{C}$, held for $4 \mathrm{~min}$, ramped at $20^{\circ} \mathrm{C} / \mathrm{min}$ to $300^{\circ} \mathrm{C}$, and held for $4.08 \mathrm{~min}$ (total acquisition time: $25 \mathrm{~min}$ ). The transfer line and ion source temperature are kept at $290^{\circ} \mathrm{C}$ and $300^{\circ} \mathrm{C}$, respectively.

\section{Results and Discussion}

Through the application of the above-reported guidelines, the harmful effects of prenatal nicotine absorption on the autonomic nervous system were highlighted in a wide case study of sudden fetal and infant deaths. More precisely, a significantly increased incidence of hypodevelopment of important brainstem and cerebellum structures was observed in fetuses and infants of smoking mothers, compared to victims with nonsmoking mothers [6]-[11].

The defects consisted mainly in hypoplasia of the neuronal centers involved in breathing control such as the Kölliker-Fuse nucleus and the facial/parafacial complex in the pons, the pre-Bötzinger nucleus in the medulla oblongata and the intermediolateral nucleus in the upper spinal cord. These nuclei, linked together in a "respiratory network" (RN), under normal conditions, function in ordered sequences: the intermediolateral nucleus coordinates during pregnancy episodic breathing movements aimed at promoting the fetal lung maturation. At the same time the Kölliker-Fuse nucleus plays an important role as it inhibits the response of central and peripheral chemoreceptors and therefore the respiratory reflexes in utero, whilst allowing occasional respiratory activity of the intermediolateral nucleus. After birth, the Kölliker-Fuse nucleus abruptly reduces its inhi- 
bitory effects and becomes active as promoter center of breathing. In detail, the Kölliker-Fuse nucleus is able to stimulate the facial/parafacial complex and the pre-Bötzinger nucleus to trigger the first inspiratory impulse as well as those thereafter [12] [13] [14] [15]. All the RN centers, through excitatory and/or inhibitory connections, according to the need, can also coordinate the pulmonary motor responses to hematic oscillations of $\mathrm{pO}_{2}, \mathrm{pCO}_{2}$ and $\mathrm{pH}$, presumably due, in many cases, to nicotine absorption [16] [17].

In cases of maternal smoking in pregnancy, carbon monoxide, a gaseous combustion product of nicotine, may readily cross the placenta and bind to fetal hemoglobin [18] [19] [20] [21]. The consequent carboxyhemoglobin is not able to release oxygen, thus hindering the physiological development of fetal organs, especially those more susceptible to hypoxic damage, including the brain. Moreover, nicotine is one of the few lipid-soluble substances that can pass through the blood-brain barrier by passive diffusion and act directly on the expression of specific receptors which are essential for the development of the nervous system, such as the nicotinic acetylcholine receptors (nAChRs). A single $\mathrm{nAChR}$ is an integral membrane protein composed of five subunits, with a central small ionic pore which normally opens when binding to the ACh agonist and closes in the resting state. The five subunits are combined to form two main receptor groups: the "homomeric receptors" containing only $\alpha$ subunits $(\alpha 7-\alpha 9)$ and the "heteromeric receptors" that contain two or more $\alpha$ and $\beta$ subtypes in the same complex ( $\alpha 2-\alpha 6$ and $\beta 2-\beta 4$ ) [22] [23] [24] [25].

Nicotine behaves as an ACh antagonist. In particular it elicits its toxic effects above all on the $\alpha 7$-nAChR subunit, due to its faster kinetics compared with other nAChR subtypes and its feature of primary target for neurotoxicants [26] [27]. So, ACh cannot perform its normal function, since the corresponding receptors are made unavailable by nicotine. As results, a significant synaptic turnover disruption and severe defects of the nervous system developmental process can happen.

In one of our studies [28] we compared the expression of $\alpha 7$-nAChRs in the brainstems of 23 fetuses and 22 newborns who died of known and unknown causes, with both smoking and nonsmoking mothers, in order to assess the possible relationship between hyperactivation of these receptors caused by nicotine absorption during pregnancy and sudden unexplained perinatal death. An overexpression of $\alpha 7$-nAChRs, associated to developmental alterations of brainstem structures involved in the breathing control (mainly hypoplasia of the parafacial/facial complex and the pre-Bötzinger and the Kölliker-Fuse nuclei), was highlighted in many SIDS and SIUDS victims with smoking mothers.

To date several Authors have focused on the effects of maternal smoking in pregnancy on the nicotinic receptors of infants who died of SIDS but with conflicting results. While Duncan et al. [29] and Nachmanoff et al. [30] did not find any differences in the receptor expression in brainstem nuclei in SIDS cases both exposed and not exposed to tobacco smoke, Macahalani et al. [31] reported intense $a 7$-nAChRs immunostaining in several brainstem nuclei in SIDS cases 
with smoking mothers, according to the results of our study [28]. Then, we can sustain that nicotine is able to perturb the structural maturation of brainstem nuclei by impairing the central cholinergic system through the upregulation of nicotinic receptor bindings. This hypothesis is also supported by experimental studies showing that stimulation of nAChRs by nicotine causes neuronal inhibition of DNA synthesis, mitotic abnormalities and apoptosis of brain structures essential for life, thus compromising their normal development [32] [33].

Many SIUDS and SIDS cases included in our study [28] with hypoplasia of one or more brainstem nuclei and high percentage of $\alpha 7$-nAChR immunopositive neurons, were born from non-smoking mothers. Therefore, we speculated that other environmental risk factors besides tobacco smoke, such as persistent pollutants, could have caused these alterations. We then used appropriate chemical procedures to analyze the cerebral cortex samples of these cases in order to evaluate the possible presence of agricultural pesticides that were used in the area of the mothers' residence, especially the endocrine disrupting compounds (EDCs), which are organic chemicals characterized by high degradation resistance and ability to interfere with the functionality of the endocrine system.

The chemical analysis, through gas chromatography-mass spectrometry (GC-MS) [34] [35], showed the presence of EDCs such as organochlorine and organophosphate pesticides (more precisely $\alpha$ and $\gamma$-chlordane, chlorfenvinfos, chlorpyrifos, $\mathrm{p}, \mathrm{p}$-DDT, $\mathrm{p}, \mathrm{p}$-DDE, endrin, $\alpha$ - and $\beta$-endosulfans) in the brain samples. The exposure to persistent EDCs in pregnancy most likely produces the same effect of nicotine on $\alpha 7$-nAChR expression and functionality. Likewise to nicotine, pesticides, when absorbed by the mothers, can pass through the placental barrier into the fetal blood stream and then, through the blood-brain barrier, into the fetal brain. Here pesticides may interfere with the expression of genes controlling the nervous system development and cause specific molecular alterations in the DNA, RNA and antigenic proteins of the neurons [36] [37].

Our findings indicate that $\alpha 7$-nAChRs are specific targets in the developing brain for nicotine as well as for cholinergic neurotoxicants, including EDCs. Therefore, prenatal absorption of cigarette smoke and pesticide, individually or together, can perturb the expression of $\alpha 7$-nAChRs in the brain of fetuses and newborns so allowing developmental alterations of neuronal centers that are essential for life. These results show that the altered expression of $\alpha 7$-nAChRs due to exposure to toxins in utero increases the risk of SIDS/SIUDS.

Nicotinic receptors are present not only in the central nervous system but also in other human tissues and organs with the same susceptibility to toxic substances [38] [39]. We have reported the presence of high $\alpha 7$-nAChR immunopositivity in the lung parenchyma and airway vessel walls in many SIUDS/SIDS victims with smoking mother [40]. This article emphasizes the extreme vulnerability of the developing lung to maternal cigarette smoke absorption, as also reported by the literature [41] [42] [43]. So, the pulmonary hypoplasia in fetuses and many respiratory disorders in infancy may be linked to the interaction of xenobiotics, including pesticides, with the lung $\alpha 7$-nAChRs. 


\section{Conclusion}

A multitude of research studies have highlighted the presence of developmental disorders of brain structures which play an important role in the control of vital functions, especially in fetal and infant deaths, associated to maternal exposure to environmental risk factors. This article, summarizing the research carried out at the Lino Rossi Center of the University of Milan in this field, adds new information on the potentially dangerous effects of cigarette smoke and air pollutants on the functionality of the nicotinic receptors during the development not only of the nervous system but also of the lungs. It follows the need to warn pregnant women that cigarette smoking places their unborn children in severe danger for life. In addition, they should not only avoid smoking but also EDC exposure whenever possible.

\section{Acknowledgements}

This study was supported by the Italian Health's Ministry in accordance with the Law 31/2006 "Regulations for Diagnostic Post Mortem Investigation in Victims of Sudden Infant Death Syndrome (SIDS) and Unexpected Fetal Death".

\section{Author Contribution}

A.M.L. conducted the research summarized here, planned and wrote the present article.

\section{Conflicts of Interest}

The author declares no conflict of interest.

\section{References}

[1] Constitution of the Italian Republic Law No. 31 (2006) Regulations for Diagnostic Post-Mortem Investigation in Victims of Sudden Infant Death Syndrome (SIDS) and Unexpected Fetal Death. Official Gazette of the Italian Republic, General Series No. 34, 4. http://users.unimi.it/centrolinorossi/files/gazz_ufficiale.pdf

[2] Roncati, L., Piscioli, F., Pusiol, T. and Lavezzi, A.M. (2017) Neuropathological Protocol for the Study of Unexplained Stillbirth. Folia Neuropathologica, 5, 79-85. https://doi.org/10.5114/fn.2017.68576

[3] Matturri, L., Ottaviani, G. and Lavezzi, A.M. (2008) Guidelines for Neuropathologic Diagnostics of Perinatal Unexpected Loss and Sudden Infant Death Syndrome (SIDS): A Technical Protocol. Virchows Archiv, 452, 19-25. https://doi.org/10.1007/s00428-007-0527-Z

[4] Lavezzi, A.M., Piscioli, F., Pusiol, T., Jorizzo, G. and Ferrero, S. (2019) Sudden Intrauterine Unexplained Death: Time to Adopt Uniform Postmortem Investigative Guidelines? BMC Pregnancy Childbirth, 19, 526. https://doi.org/10.1186/s12884-019-2603-1

[5] Alfonsi, G. and Crippa, M. (2016) Updates on the Methodological Approaches for Carrying Out an In-Depth Study of the Cardiac Conduction System and the Autonomic Nervous System of Victims of Sudden Unexplained Fetal and Infant Death. Frontiers of Medicine (Lausanne), 3, 54. https://doi.org/10.3389/fmed.2016.00054 
[6] Lavezzi, A.M., Ottaviani, G., Mauri, M. and Matturri, L. (2007) Biopathology of the Dentate-Olivary Complex in Sudden Unexplained Perinatal Death and Sudden Infant Death Syndrome Related to Maternal Cigarette Smoking. Neurological Research, 29, 525-532. https://doi.org/10.1179/016164107X166308

[7] Lavezzi, A.M., Matturri, L., Del Corno, G. and Johanson, C.E. (2013) Vulnerability of Fourth Ventricle Choroid Plexus in Sudden Unexplained Fetal and Infant Death Syndromes Related to Smoking Mothers. International Journal of Developmental Neuroscience, 31, 319-327. https://doi.org/10.1016/j.ijdevneu.2013.04.006

[8] Lavezzi, A.M., Corna, M.F. and Matturri, L. (2010) Ependymal Alterations in Sudden Intrauterine Unexplained Death and Sudden Infant Death Syndrome: Possible Primary Consequence of Prenatal Exposure to Cigarette Smoking. Neural Development, 5, 17. https://doi.org/10.1186/1749-8104-5-17

[9] Lavezzi, A.M., Corna, M.F., Repetti, M.L. and Matturri, L. (2013) Cerebellar Purkinje Cell Vulnerability to Prenatal Nicotine Exposure in Sudden Unexplained Perinatal Death. Folia Neuropathologica, 51, 290-301.

https://doi.org/10.5114/fn.2013.39718

[10] Lavezzi, A.M., Mecchia, D. and Matturri, L. (2012) Neuropathology of the Area Postrema in Sudden Intrauterine and Infant Death Syndromes Related to Tobacco Smoke Exposure. Autonomic Neuroscience: Basic and Clinical, 166, 29-34. https://doi.org/10.1016/j.autneu.2011.09.001

[11] Lavezzi, A.M., Ottaviani, G. and Matturri, L. (2005) Adverse Effects of Prenatal Tobacco Smoke Exposure on Biological Parameters of the Developing Brainstem. Neurobiology of Disease, 20, 601-607. https://doi.org/10.1016/j.nbd.2005.04.015

[12] Lavezzi, A.M., Ottaviani, G., Rossi, L. and Matturri, L. (2004) Cytoarchitectural Organization of the Parabrachial/Kölliker-Fuse Complex in Man. Brain and Development, 26, 316-320. https://doi.org/10.1016/j.braindev.2003.09.002

[13] Lavezzi, A.M. and Matturri, L. (2008) Hypoplasia of the Parafacial/Facial Complex: A Very Frequent Finding in Sudden Unexplained Fetal Death. The Open Neuroscience Journal, 2, 1-5. https://doi.org/10.2174/1874082000802010001

[14] Lavezzi, A.M. and Matturri, L. (2008) Functional Neuroanatomy of the Human Pre-Bötzinger Complex with Particular Reference to Sudden Unexplained Perinatal and Infant Death. Neuropathology, 28, 10-16. https://doi.org/10.1111/j.1440-1789.2007.00824.x

[15] Lavezzi, A.M., Corna, M.F., Mehboob, R. and Matturri, L. (2009) Neuropathology of the Intermediolateral Nucleus of the Spinal Cord in Sudden Unexplained Perinatal and Infant Death of Raphe Nuclei and Serotonin Transporter Gene Promoter Polymorphism. Pediatric Research, 66, 22-27.

https://doi.org/10.1203/PDR.0b013e3181a7bb73

[16] Lavezzi, A.M., Ferrero, S., Matturri, L., Roncati, L. and Pusiol, T. (2016) Developmental Neuropathology of Brainstem Respiratory Centers in Unexplained Stillbirth: What's the Meaning? International Journal of Developmental Neuroscience, 53, 99-106. https://doi.org/10.1016/j.ijdevneu.2016.06.007

[17] Lavezzi, A.M. (2015) A New Theory to Explain the Underlying Pathogenetic Mechanism of Sudden Infant Death Syndrome. Frontiers in Neurology, 6, 220.

[18] Prockop, L.D. and Chichkova, R.I. (2007) Carbon Monoxide Intoxication: An Updated Review. Journal of the Neurological Sciences, 262, 122-130. https://doi.org/10.1016/j.jns.2007.06.037

[19] Longo, L.D. (1982) Some Health Consequences of Maternal Smoking: Issues without Answers. Birth Defects, 18, 13-31. 
[20] Okeda, R., Matsuo, T., Kuroiwa, T., Tajima, T. and Takahashi, H. (1986) Experimental Study on Pathogenesis of the Fetal Brain Damage by Acute Carbon Monoxide Intoxication of the Pregnant Mother. Acta Neuropathologica (Berl.), 69, 244-252. https://doi.org/10.1007/BF00688300

[21] Lichtensteiger, W., Ribary, U., Schiumpf, M., Odermatt, B. and Widemer, H.R. (1988) Prenatal Adverse Effects of Nicotine on the Developing Brain. Progress in Brain Research, 73, 137-157. https://doi.org/10.1016/S0079-6123(08)60502-6

[22] Picciotto, M.R., Higley, M.J. and Mineur, Y.S. (2012) Acetylcholine as a Neuromodulator: Cholinergic Signaling Shapes Nervous System Function and Behavior. Neuron, 76, 116. https://doi.org/10.1016/j.neuron.2012.08.036

[23] Boyd, R.T. (1997) The Molecular Biology of Neuronal Nicotinic Acetylcholine Receptors. Critical Reviews in Toxicology, 27, 299-318.

[24] Lindstrom, J., Anand, R., Gerzanich, V., Peng, X., Wang, F. and Wells, G. (1996) Structure and Function of Neuronal Nicotinic Acetylcholine Receptors. Progress in Brain Research, 109, 125-137. https://doi.org/10.1016/S0079-6123(08)62094-4

[25] Albuquerque, E.X., Pereira, E.F., Alkondon, M. and Rogers, S.W. (2009) Mammalian Nicotinic Acetylcholine Receptors: From Structure to Function. Physiological Reviews, 89, 73-120. https://doi.org/10.1152/physrev.00015.2008

[26] Broide, R.S. and Leslie, F.M. (1999) The alpha7 Nicotinic Acetylcoline Receptor in Neuronal Plasticity. Molecular Neurobiology, 20, 1-16.

[27] Falk, L., Nordberg, A., Seiger, A., Kjaeldgaard, A. and Hellström-Lindahl, E. (2002) The alpha7 Nicotinic Receptors in Human Fetal Brain and Spinal Cord. Journal of Neurochemistry, 80, 457-465.

[28] Lavezzi, A.M., Cappiello, A., Pusiol, T., Corna, M.F., Termopoli, V. and Matturri, L. (2015) Pesticide Exposure during Pregnancy, Like Nicotine, Affects the Brainstem a7 Nicotinic Acetylcholine Receptor Expression, Increasing the Risk of Sudden Unexplained Perinatal Death. Journal of the Neurological Sciences, 348, 94-100. https://doi.org/10.1016/j.jns.2014.11.014

[29] Duncan, J.R., Welty, T.K., Iyasu, S. and Kinney, H.C. (2008) The Effect of Maternal Smoking and Drinking during Pregnancy upon (3)H-nicotine Receptor Brainstem Binding in Infants Dying of the Sudden Infant Death Syndrome: Initial Observations in a High Risk Population. Brain Pathology, 18, 21-31. https://doi.org/10.1111/j.1750-3639.2007.00093.x

[30] Nachmanoff, D.B., Panigrahy, A., Filiano, J.J., Mandell, F., Sleeper, L.A. Valdes-Dapena, M., Krous, H.F., White, W.F. and Kinney, H.C. (1998) Brainstem $3 \mathrm{H}$-Nicotine Receptor Binding in the Sudden Infant Death Syndrome. Journal of Neuropathology \& Experimental Neurology, 57, 1018-1025.

https://doi.org/10.1097/00005072-199811000-00004

[31] Machaalani, R., Say, M. and Waters, K.A. (2011) Effects of Cigarette Smoke Exposure on Nicotinic Acetylcholine Receptor Subunits $\alpha 7$ and $\beta 2$ in the Sudden Infant Death Syndrome (SIDS) Brainstem. Toxicology and Applied Pharmacology, 257, 396-404. https://doi.org/10.1016/j.taap.2011.09.023

[32] Atluri, P., Fleck, M.W., Shen, Q., Mah, S.J., Stadfelt, D., Barnes, W., Goderie, S.K., Temple, S. and Schneider, A.S. (2001) Functional Nicotinic Acetylcholine Receptor Expression in Stem and Progenitor Cells of the Early Embryonic Mouse Cerebral Cortex. Developmental Biology, 240, 143-156.

https://doi.org/10.1006/dbio.2001.0453

[33] Roy, T.S., Andrews, J.E., Seidler, F.J. and Slotkin, T.A. (1998) Nicotine Evokes Cell Death in Embryonic Rat Brain during Neurulation. Journal of Pharmacology and 
Experimental Therapeutics, 287, 1135-1144.

[34] Cappiello, A., Famiglini, G., Palma, P., Termopoli, V., Lavezzi, A.M. and Matturri, L. (2014) Determination of Selected Endocrine Disrupting Compounds in Human Fetal and Newborn Tissues by GC-MS. Analytical and Bioanalytical Chemistry, 406, 2779-2788.

[35] Pusiol, T., Lavezzi, A.M., Matturri, L., Termopoli, V., Cappiello, A., Piscioli, F. and Roncati, L. (2016) Impact Assessment of Endocrine Disruptors on Sudden Intrauterine and Infant Death Syndromes. European Journal of Forensic Sciences, 3, 8-15. https://doi.org/10.5455/ejfs.197968

[36] Roncati, L., Termopoli, V. and Pusiol, T. (2016) Negative Role of the Environmental Endocrine Disruptors in the Human Neurodevelopment. Frontiers in Neurology, 7, 143.

[37] Roncati, L., Piscioli, F. and Pusiol, T. (2016) The Endocrine Disruptors among the Environmental Risk Factors for Stillbirth. Science of the Total Environment, 563-564, 1086-1087. https://doi.org/10.1016/j.scitotenv.2016.04.214

[38] Wessler, I. and Kirkpatrick, C.J. (2008) Acetylcholine beyond Neurons: The Non-Neuronal Cholinergic System in Humans. British Journal of Pharmacology, 154, 1558-1571. https://doi.org/10.1038/bjp.2008.185

[39] Spindel, E.R. (2003) Neuronal Nicotinic Acetylcholine Receptors: Not Just in Brain. American Journal of Physiology-Lung Cellular and Molecular Physiology, 285, 1201-1202. https://doi.org/10.1152/ajplung.00251.2003

[40] Lavezzi, A.M., Corna, M.F., Alfonsi, G. and Matturri, L. (2014) Possible Role of the $\alpha 7$ Nicotinic Receptors in Mediating Nicotine's Effect on Developing Lung-Implications in Unexplained Human Perinatal Death. BMC Pulmonary Medicine, 14, 11. https://doi.org/10.1186/1471-2466-14-11

[41] Fu, X.W., Lindstrom, J. and Spindel, E.R. (2009) Nicotine Activates and Up-Regulates Nicotinic Acetylcholine Receptors in Bronchial Epithelial Cells. American Journal of Physiology_Lung Cellular and Molecular Physiology, 41, 93-99. https://doi.org/10.1165/rcmb.2008-0352OC

[42] Sekhon, H.S., Jia, Y., Raab, R., Kuryatov, A., Pankow, J.F., Whitsett, J.A., Lindstrom, J. and Spindel, E.R. (1999) Prenatal Nicotine Increases Pulmonary alpha7 Nicotinic Receptor Expression and Alters Fetal Lung Development in Monkeys. Journal of Clinical Investigation, 103, 637-647. https://doi.org/10.1172/JCI5232

[43] Sekhon, H.S., Keller, J.A., Benowitz, N.L. and Spindel, E.R. (2001) Prenatal Nicotine Exposure Alters Pulmonary Function in Newborn Rhesus Monkeys. American Journal of Respiratory and Critical Care Medicine, 164, 989-994.

https://doi.org/10.1164/ajrccm.164.6.2011097 